\title{
Editorial: Steroids and Secosteroids in the Modulation of Inflammation and Immunity
}

\author{
Andrzej T. Slominski ${ }^{1,2 *}$, Bidesh Mahata ${ }^{3 *}$, Chander Raman ${ }^{1 *}$ and Oxana Bereshchenko ${ }^{4 *}$ \\ ${ }^{1}$ Department of Dermatology, University of Alabama at Birmingham, Birmingham, AL, United States, ${ }^{2}$ Pathology Laboratory \\ Service, Veteran Administration Medical Center, Birmingham, AL, United States, ${ }^{3}$ Department of Pathology, University of \\ Cambridge, Cambridge, United Kingdom, ${ }^{4}$ Department of Philosophy, Social Sciences and Education, University of Perugia, \\ Perugia, Italy
}

Keywords: steroids, secosteroids, steroidogenesis, secosteroidogenesis, Inflammation, immunity, vitamin D, glucocorticoids

\section{Editorial on the Research Topic}

\section{OPEN ACCESS}

Edited and reviewed by: Silvano Sozzani,

Sapienza University of Rome, Italy

${ }^{*}$ Correspondence: Andrzej T. Slominski aslominski@uabmc.edu Bidesh Mahata

bm562@cam.ac.uk

Chander Raman

chanderraman@uabmc.edu

Oxana Bereshchenko oxana.bereshchenko@unipg.it

Specialty section:

This article was submitted to Cytokines and Soluble Mediators in Immunity, a section of the journal

Frontiers in Immunology

Received: 30 November 2021 Accepted: 06 December 2021 Published: 20 December 2021

Citation:

Slominski AT, Mahata B, Raman $C$ and Bereshchenko $O$ (2021) Editorial: Steroids and Secosteroids in the Modulation of Inflammation and Immunity.

Front. Immunol. 12:825577. doi: 10.3389/fimmu.2021.825577
Steroids and Secosteroids in the Modulation of Inflammation and Immunity

In this Research Topic of Frontiers in Immunology focused on the steroids and secosteroids in the modulation of inflammation and immunity 11 articles by experts in corresponding fields have been published (Bier et al.; Bruscoli et al.; He et al.; König et al.; Lucafò et al.; Merk et al.; Postlethwaite et al.; Quatrini et al.; Shimba et al.; Vanderhaeghen et al.; Xie et al.). These included research articles, reviews and mini-reviews papers on important aspects of steroid- and secosteroidogenesis and the role of the final or intermediate products of these pathways in regulation of inflammatory and immune activities. Mechanisms of action and of broad homeostatic activities in humans and experimental animal models have also been discussed in these expert written papers. Different aspects of biochemistry, molecular biology, cell biology, and the systems-level role of (seco) steroidogenesis in regulating physiology and pathology have been discussed.

The key role in steroidogenesis is played by an enzyme CYP11A1, a member of the cytochrome P450 family, which catalyzes the first and rate-limiting step in steroidogenesis, converting cholesterol to pregnenolone through sequential its hydroxylation at $\mathrm{C} 22$ and $\mathrm{C} 20$, with a final cleavage of the side chain (1-4). In addition to the adrenals, gonads and placenta (classical steroidogenic tissues), CYP11A1 is also expressed in the brain (6), gastrointestinal tract, immune systems (7-9), the skin (5) and other peripheral organs/tissues $(10)$ including malignant tumors $(9,11)$. The roles of local steroidogenesis (i.e., extraglandular steroidogenesis, including immune cell mediated steroidogenesis) are emerging and warrant a revisit to this important biosynthetic pathway and its functional involvement in tissue homeostasis (including immune homeostasis) and disease $(7,12,13)$. Recent discoveries pointed out an important, and unexpected role for CYP11A1 in metabolism of 7-dehydrocholestrol (14), vitamin D3 (15), D2 (16), lumisterol $(17,18)$ and ergosterol $(11)$ to several biologically active metabolites. Thus, CYP11A1 activity is opening several novel pathways generating $\Delta 7$ steroids, full chain and short chain lumisterol derivatives, and secosteroids (vitamin $\mathrm{D}$ hydroxyderivatives) $(5,19)$. While the biological significance of these pathways is currently being evaluated, it must be noted that CYP11A1-derived hydroxyderivatives of vitamin $\mathrm{D}(20,21)$ and of lumisterol $(18)$ are circulating in human serum and 
are detectable in the epidermis. Furthermore, recent data showing that 7-Dehydrocholesterol reductase (DHCR7, which catalyzes the reduction of the $\mathrm{C} 7-\mathrm{C} 8$ double bond of its B-ring that is necessary for the final formation of cholesterol) did not act on 7 dehydropregnenolone and lumisterol compounds (22), enhances the importance of pathways generating $\Delta 7$ steroids and lumisterol derivatives $(20,23,24)$.

As relates to secosteroids, in this Research Topic, a paper by Postlethwaite et al. reports that CYP11A1-derived 20Shydroxyvitamin D3 [20S(OH)D3] markedly suppresses clinical signs of arthritis and joint damage in a mouse model of rheumatoid arthritis (RA). 20S(OH)D3 also changes proportion of lymphocyte subsets in peripheral blood resulting in a significant reduction in the levels of inflammatory cytokines, and decrease in complement-fixing anti-CII antibodies. The authors propose further consideration for $20 \mathrm{~S}(\mathrm{OH}) \mathrm{D} 3$ in treatment of RA and other autoimmune disorders (Postlethwaite et al.).
Concerning extra-adrenal glucocorticoids synthesis, Merk et al. review a role of epithelial barriers as alternative routes for their synthesis at the local and perhaps systemic levels. Their role in the inter-organ communication through an interconnected crosstalk that counteract pro-inflammatory activities and prevent autoimmune activities are discussed (Merk et al.). These considerations are in line with similar concepts previously proposed $(7,13,25,26)$ and are consistent with communication between peripheral and central endocrine regulators (27) including hypothalamo-pituitary-adrenal axis (Figure 1) $(7,28$, 29). Therefore, regulation of local corticosteroidogenesis may serve as a viable therapeutic alternative to using synthetic corticosteroids in the therapy of inflammatory or autoimmune disorders (Merk et al.; 7; 13). Such regulation can be achieved by using specific wavelength of ultraviolet radiation (UVR) (30).

Of similar importance are three other mini-reviews and two reviews. One on regulation of the immune system development by

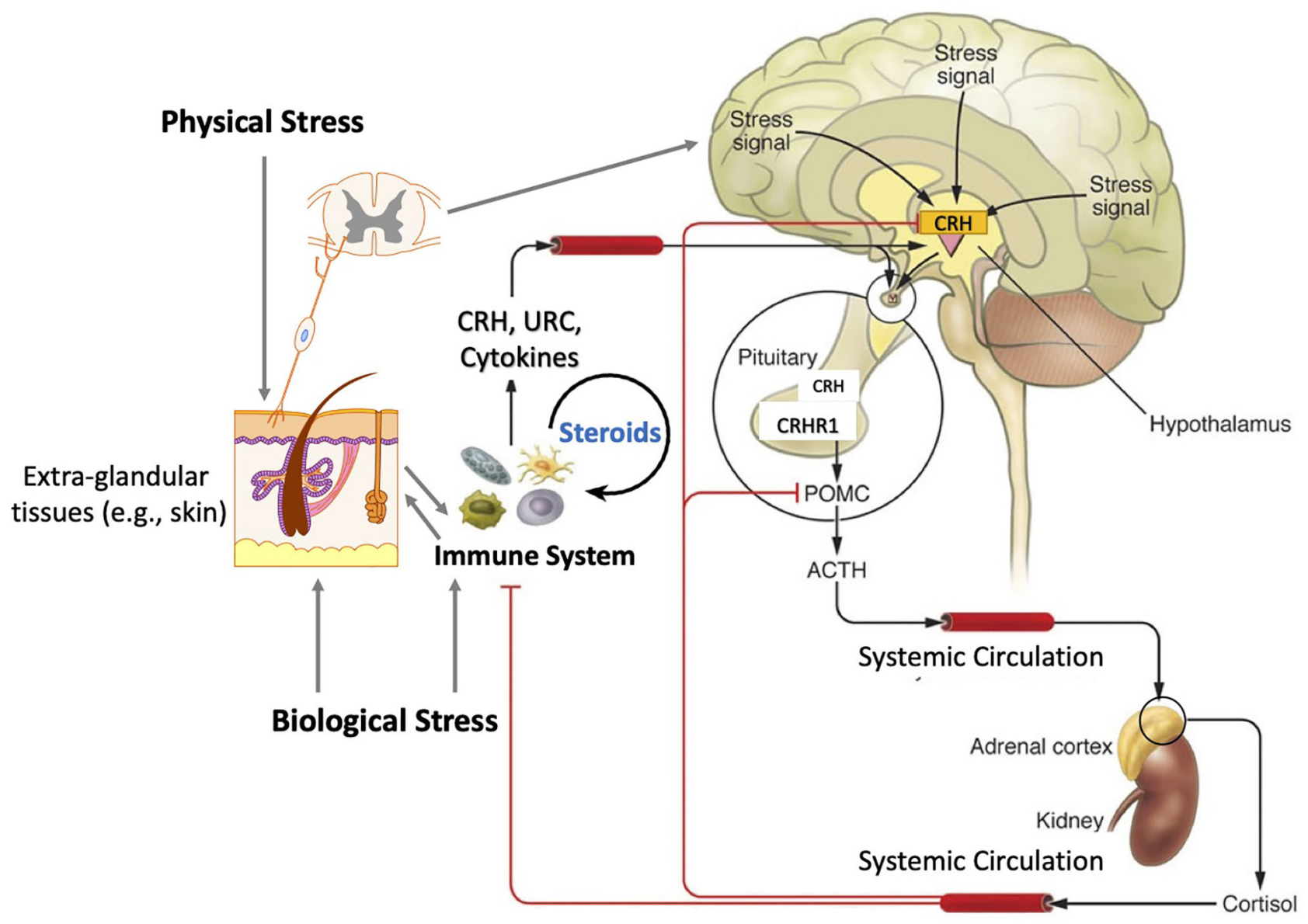

FIGURE 1 | The functional organization of the hypothalamic-pituitary-adrenal axis with inputs from the immune system and peripheral organs. Physical and biological stress promotes the release of stress signals in both the brain, the skin, and immune cells, resulting in the hypothalamic release of corticotropin releasing hormone (CRH), which in turn stimulates the release of adrenocorticotropic hormone (ACTH) and pro-opiomelanocortin (POMC) expression and processing in the anterior pituitary. ACTH binds to the melanocortin-2 (MC2) receptor in the zona fasciculata of the adrenal cortex and stimulates the transport of cholesterol into the mitochondria and stimulates the production cortisol. Glucocorticoids not only regulate body homeostasis but also act in a negative feedback loop for CRH and POMC expression. Immune cell-mediated steroidogenesis and secosteroidogenesis are emerging as new modes of immune regulation. Reprinted with permission from the $(7,28)$. 
glucocorticoids and sex hormones (Quatrini et al.), which also include control of the hematopoietic stem cell differentiation and subsequent maturation of immune cell subsets. Second mini-review (Bruscoli et al.) discusses glucocorticoid therapy in inflammatory bowel diseases with consideration of new glucocorticoids mediators, such as glucocorticoid-induced leucine zipper, which may have similar anti-inflammatory properties. The third mini-review (Shimba et al.) discusses pleiotropic effects of glucocorticoids on the Immune system in the context of circadian rhythm and stress. Among two reviews one (He et al.) analyses glucocorticoid-induced leucine zipper as a promising marker for monitoring and treating sepsis. The second one (Vanderhaeghen et al.) discusses bidirectional crosstalk between hypoxia inducible factors and glucocorticoid signaling in health and disease, being in line with a fascinating subject of linking immune activity with immune cells energy yielding metabolism (31).

The four original research papers discuss important experimental evidences including use of IFN $\gamma / \mathrm{IL} 10$ ratio for stratification of hydrocortisone (a synthetic but identical molecule to endogenous cortisol) therapy in patients with septic shock (König et al.), glucocorticoid-induced exacerbation of mycobacterial infection through a reduced phagocytic capacity of macrophages (Xie et al.), protection of antigen-primed effector $\mathrm{T}$ cells from glucocorticoid-induced apoptosis in cell culture and in a mouse model of multiple sclerosis (Bier et al.), and that gender may influence the immunosuppressive actions of prednisone (a synthetic glucocorticoids with much higher potency than cortisol) in inflammatory bowel disease (Lucafò et al.).

Thus, this Research Topic discusses several important aspects of steroidogenesis, secosteroidogenesis, and their role in cell signaling cascades in the context of physiology and pathology of inflammation and immunity. One important conclusion is that the deregulation of steroidogenic and secosteroidogenic signaling pathways may also lead to a variety of inflammatory disorders and autoimmune diseases in a gender- and contextdependent manner. Different therapeutic and preventive strategies can be deducted from the presented papers leading to practical and clinical solutions to many inflammatory and autoimmune diseases. In future, further analytical investigation is required to understand the physiological and pathological role of endogenous steroids and secosteroids.

\section{AUTHOR CONTRIBUTIONS}

AS wrote the first-draft and others (BM, CR, OB) contributed to improve the writing and conceptual Figures. Correspondence can be made to anyone or all of these authors (AS, BM, CR, OB). All authors contributed to the article and approved the submitted version.

\section{FUNDING}

Writing of this editorial was supported by NIH grants R01AR073004, R01AR071189, R21 AI149267, AR069010, AR064825, R21AR063242, VA merit grant 1I01BX004293-01A1. BM is supported by CRUK Career Development Fellowship (RCCFEL\100095), NSF-BIO/UKRI-BBSRC project grant (BB/ V006126/1) and MRC project grant (MR/V028995/1).

\section{REFERENCES}

1. Miller WL. Steroid Hormone Synthesis in Mitochondria. Mol Cell Endocrinol (2013) 379:62-73. doi: 10.1016/j.mce.2013.04.014

2. Strushkevich N, MacKenzie F, Cherkesova T, Grabovec I, Usanov S, Park HW. Structural Basis for Pregnenolone Biosynthesis by the Mitochondrial Monooxygenase System. Proc Natl Acad Sci USA (2011) 108:10139-43. doi: 10.1073/pnas.1019441108

3. Goursaud C, Mallet D, Janin A, Menassa R, Tardy-Guidollet V, Russo G, et al. Aberrant Splicing Is the Pathogenicity Mechanism of the P.Glu314Lys Variant in CYP11A1 Gene. Front Endocrinol (Lausanne) (2018) 9:491. doi: 10.3389/fendo.2018.00491

4. Tuckey RC. Progesterone Synthesis by the Human Placenta. Placenta (2005) 26:273-81. doi: 10.1016/j.placenta.2004.06.012

5. Slominski RM, Raman C, Elmets C, Jetten AM, Slominski AT, Tuckey RC. The Significance of CYP11A1 Expression in Skin Physiology and Pathology. Mol Cell Endocrinol (2021) 530:111238. doi: 10.1016/j.mce.2021.111238

6. Lin YC, Papadopoulos V. Neurosteroidogenic Enzymes: CYP11A1 in the Central Nervous System. Front Neuroendocrinol (2021) 62:100925. doi: 10.1016/j.yfrne.2021.100925

7. Slominski RM, Tuckey RC, Manna PR, Jetten AM, Postlethwaite A, Raman C, et al. Extra-Adrenal Glucocorticoid Biosynthesis: Implications for Autoimmune and Inflammatory Disorders. Genes Immun (2020) 21:15068. doi: 10.1038/s41435-020-0096-6

8. Mahata B, Zhang X, Kolodziejczyk AA, Proserpio V, Haim-Vilmovsky L, Taylor AE, et al. Single-Cell RNA Sequencing Reveals T Helper Cells Synthesizing Steroids De Novo to Contribute to Immune Homeostasis. Cell Rep (2014) 7:1130-42. doi: 10.1016/j.celrep.2014.04.011

9. Mahata B, Pramanik J, van der Weyden L, Polanski K, Kar G, Riedel A, et al. Tumors Induce De Novo Steroid Biosynthesis in T Cells to Evade Immunity. Nat Commun (2020) 11:3588. doi: 10.1038/s41467-020-17339-6

10. Slominski AT, Li W, Kim TK, Semak I, Wang J, Zjawiony JK, et al. Novel Activities of CYP11A1 and Their Potential Physiological Significance. J Steroid Biochem Mol Biol (2015) 151:25-37. doi: 10.1016/j.jsbmb.2014.11.010

11. Slominski A, Semak I, Zjawiony J, Wortsman J, Gandy MN, Li J, et al. Enzymatic Metabolism of Ergosterol by Cytochrome P450scc to Biologically Active 17alpha,24-Dihydroxyergosterol. Chem Biol (2005) 12:931-9. doi: 10.1016/j.chembiol.2005.06.010

12. Chakraborty S, Pramanik J, Mahata B. Revisiting Steroidogenesis and its Role in Immune Regulation With the Advanced Tools and Technologies. Genes Immun (2021) 22:125-40. doi: 10.1038/s41435-021-00139-3

13. Slominski A, Zbytek B, Nikolakis G, Manna PR, Skobowiat C, Zmijewski M, et al. Steroidogenesis in the Skin: Implications for Local Immune Functions. J Steroid Biochem Mol Biol (2013) 137:107-23. doi: 10.1016/j.jsbmb.2013.02.006

14. Slominski AT, Kim TK, Chen J, Nguyen MN, Li W, Yates CR, et al. Cytochrome P450scc-Dependent Metabolism of 7-Dehydrocholesterol in Placenta and Epidermal Keratinocytes. Int J Biochem Cell Biol (2012) 44:2003-18. doi: 10.1016/j.biocel.2012.07.027

15. Slominski AT, Kim TK, Shehabi HZ, Semak I, Tang EK, Nguyen MN, et al. In Vivo Evidence for a Novel Pathway of Vitamin D(3) Metabolism Initiated by P450scc and Modified by CYP27B1. FASEB J (2012) 26:3901-15. doi: 10.1096/ fj.12-208975

16. Slominski AT, Kim TK, Shehabi HZ, Tang EK, Benson HA, Semak I, et al. In Vivo Production of Novel Vitamin D2 Hydroxy-Derivatives by Human Placentas, Epidermal Keratinocytes, Caco-2 Colon Cells and the Adrenal Gland. Mol Cell Endocrinol (2014) 383:181-92. doi: 10.1016/j.mce.2013.12.012 
17. Tuckey RC, Slominski AT, Cheng CY, Chen J, Kim TK, Xiao M, et al. Lumisterol is Metabolized by CYP11A1: Discovery of a New Pathway. Int J Biochem Cell Biol (2014) 55:24-34. doi: 10.1016/j.biocel.2014.08.004

18. Slominski AT, Kim TK, Hobrath JV, Janjetovic Z, Oak ASW, Postlethwaite A, et al. Characterization of a New Pathway That Activates Lumisterol In Vivo to Biologically Active Hydroxylumisterols. Sci Rep (2017) 7:11434. doi: 10.1038/ s41598-017-10202-7

19. Slominski AT, Chaiprasongsuk A, Janjetovic Z, Kim TK, Stefan J, Slominski RM, et al. Photoprotective Properties of Vitamin D and Lumisterol Hydroxyderivatives. Cell Biochem Biophys (2020) 78:165-80. doi: 10.1007/ s12013-020-00913-6

20. Jenkinson C, Desai R, Slominski AT, Tuckey RC, Hewison M, Handelsman DJ. Simultaneous Measurement of 13 Circulating Vitamin D3 and D2 Mono and Dihydroxy Metabolites Using Liquid Chromatography Mass Spectrometry. Clin Chem Lab Med (2021) 59(10):1642-52. doi: 10.1515/ cclm-2021-0441

21. Slominski AT, Kim TK, Li W, Postlethwaite A, Tieu EW, Tang EK, et al. Detection of Novel CYP11A1-Derived Secosteroids in the Human Epidermis and Serum and Pig Adrenal Gland. Sci Rep (2015) 5:14875. doi: 10.1038/ srep 14875

22. Tuckey RC, Tang EKY, Chen YA, Slominski AT. Selective Ability of Rat 7Dehydrocholesterol Reductase (DHCR7) to Act on Some 7-Dehydrocholesterol Metabolites But Not on Lumisterol Metabolites. J Steroid Biochem Mol Biol (2021) 212:105929. doi: 10.1016/j.jsbmb.2021.105929

23. Slominski AT, Kim TK, Qayyum S, Song Y, Janjetovic Z, Oak ASW, et al. And Lumisterol Derivatives can Act on Liver X Receptors (LXRs). Sci Rep (2021) 11:8002. doi: 10.1038/s41598-021-87061-w

24. Slominski A, Kim TK, Zmijewski MA, Janjetovic Z, Li W, Chen J, et al. Novel Vitamin D Photoproducts and Their Precursors in the Skin. Dermatoendocrinol (2013) 5:7-19. doi: 10.4161/derm.23938

25. Phan TS, Schink L, Mann J, Merk VM, Zwicky P, Mundt S, et al. Keratinocytes Control Skin Immune Homeostasis Through De Novo-Synthesized Glucocorticoids. Sci Adv (2021) 7. doi: 10.1126/sciadv.abe0337
26. Slominski AT, Zmijewski MA, Zbytek B, Tobin DJ, Theoharides TC, Rivier J. Key Role of CRF in the Skin Stress Response System. Endocr Rev (2013) 34:827-84. doi: 10.1210/er.2012-1092

27. Slominski A, Wortsman J. Neuroendocrinology of the Skin. Endocr Rev (2000) 21:457-87. doi: 10.1210/er.21.5.457

28. Slominski A. A Nervous Breakdown in the Skin: Stress and the Epidermal Barrier. J Clin Invest (2007) 117:3166-9. doi: 10.1172/JCI33508

29. Slominski A, Wortsman J, Tuckey RC, Paus R. Differential Expression of HPA Axis Homolog in the Skin. Mol Cell Endocrinol (2007) 265-266:143-9. doi: 10.1016/j.mce.2006.12.012

30. Slominski AT, Zmijewski MA, Plonka PM, Szaflarski JP, Paus R. How UV Light Touches the Brain and Endocrine System Through Skin, and Why. Endocrinology (2018) 159:1992-2007. doi: 10.1210/en.2017-03230

31. Pearce EL. Metabolism as a Driver of Immunity. Nat Rev Immunol (2021) 21:618-9. doi: 10.1038/s41577-021-00601-3

Conflict of Interest: The authors declare that the research was conducted in the absence of any commercial or financial relationships that could be construed as a potential conflict of interest.

Publisher's Note: All claims expressed in this article are solely those of the authors and do not necessarily represent those of their affiliated organizations, or those of the publisher, the editors and the reviewers. Any product that may be evaluated in this article, or claim that may be made by its manufacturer, is not guaranteed or endorsed by the publisher.

Copyright (c) 2021 Slominski, Mahata, Raman and Bereshchenko. This is an open-access article distributed under the terms of the Creative Commons Attribution License (CC BY). The use, distribution or reproduction in other forums is permitted, provided the original author(s) and the copyright owner(s) are credited and that the original publication in this journal is cited, in accordance with accepted academic practice. No use, distribution or reproduction is permitted which does not comply with these terms. 